\title{
Immunoadsorption combined with membrane filtration might enhance desensitization
}

The success of $\mathrm{ABO}$-incompatible (ABOi) kidney transplantation depends on the use of apheresis to eliminate $\mathrm{ABO}$-specific antibodies. In a recent study led by Georg Böhmig at the Medical University of Vienna, Austria, combined semiselective immunoadsorption (IA) and membrane filtration (MF) effectively decreased IgM titres in patients, and reduced complement activation in vitro.

"Living donor kidney transplantation across major $\mathrm{ABO}$ blood group barriers has

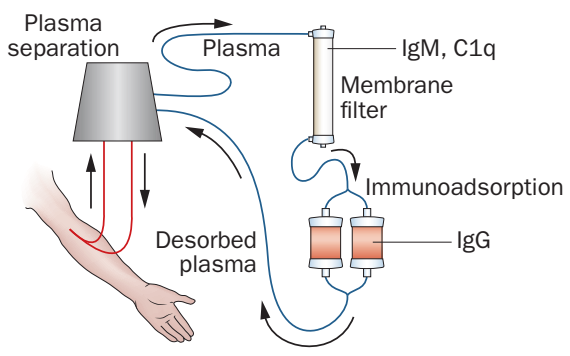

Immunoadsorption with membrane filtration. Courtesy of Georg A. Böhmig. proven an efficient way to enlarge donor pools and shorten transplant waiting times," explains Böhmig. However, the use of desensitization protocols, including antigenspecific IA, is costly and limited by $\mathrm{ABO}$ specificity. "In this respect, regenerative, semiselective, non-antigen-specific IA may offer an attractive alternative, especially because of its unselectivity regarding IgG adsorption." The researchers conducted a proof-of-concept cross-over trial to compare the effects of IA for IgG elimination alone with IA plus MF for macromolecule elimination (ABO-specific IgM) in 14 patients receiving IA for other indications.

IA plus MF treatment depleted $\mathrm{ABO}$ specific IgM - the primary end point of the study. "Perhaps our most impressive finding was a virtually complete elimination of the classical complement key component C1q," says Böhmig. Alongside a substantial influence on the functionality of complement (as assessed by the detection of antibody-mediated activation of complement component C3), these results have implications not only for ABOi transplantation, but other indications that include complement-mediated graft rejection.

The researchers plan to carry out a prospective multicentre pilot study to determine the safety and efficacy of this combined treatment in $\mathrm{ABOi}$ transplantation as an option for recipient desensitization. "Our data may provide a solid basis for future experimental and clinical research, thereby dissecting the applicability of this innovative principle," Böhmig concludes.

\section{Ellen Bible}

Original article Eskandary, F. et al. $\mathrm{ABO}$ antibody and complement depletion by immunoadsorption combined with membrane filtration-a randomized, controlled, cross-over trial. Nephrol. Dial. Transplant. doi:10.1093/ndt/gft502 